Pollard, 1978). Although further childbirth is an easily identifiable time of risk when prophylactic medication can be offered, confining lithium treatment to the post-partum period would not have prevented most of the episodes of further illness in this study.

Only two out of the nine women started on lithium continued with the medication for a long period: one had no further recorded episodes of illness; the other remained well for two years but had a further episode of illness nine months after stopping lithium treatment, so was restarted on it.

In conclusion, treatment with lithium should be considered for patients discharged following a first episode of puerperal psychosis, and there would seem to be a strong case for longer-term lithium prophylaxis.

LINDSAY, J. S. B. \& POLLARD, D. E. (1978) Mothers and children in hospital. Australian and New Zealand Journal of Psychiatry, 12 245-253.

MCKENZIE, M. H. (1991) The outcome of women with postpartum psychoses admitted to a mother and baby unit. MSc Thesis: University of Manchester.

Protheroe, C. (1969) Puerperal psychosis: a long-term study (1927-1961). British Journal of Psychiatry, 115, 9-30.

Edenfield Centre

MARTine McKenzie

Prestwich Hospital

Bury New Road

Bury

School of Psychiatry and Behavioural Sciences

University of Manchester

Rawnsley Building

Manchester Royal Infirmary

Oxford Road

Manchester M13 9WL

\section{Assessment of insight in Singapore}

SIR: The assessment of insight in psychotic patients has always been difficult, even though it is an integral part of the mental state examination. Therefore, I was highly interested in the paper by David et al (Journal, November 1992, 161, 599-602). The authors have demonstrated the value and the applicability of the schedule for assessing the components of insight (David, 1990) in psychotic patients. There are three components of this schedule (treatment compliance, recognition of illness, and ability to relabel the psychotic experience as abnormal) which David argued form the core features of insight.

The authors have sought to correlate the three components with variables such as intelligence quotient (IQ), sex, age, diagnosis, number of previous admissions, and psychopathology. They showed that the total insight score is inversely related to the psychopathology measured by the Present State Examination (PSE; Wing et al, 1974), while compliance and illness recognition correlated to IQ, but not to the other variables. However, an additional variable may influence assessment of insight (especially if one works in a multiracial society as I do in Singapore): that of cultural beliefs.

Each of the different races in Singapore (Malays, Chinese, and Indians) have their own beliefs about the abnormal behaviour and experiences which the West terms as mental illness. These beliefs include the person being possessed by evil spirits, the soul being stolen, lost or sick, and the patient being cursed or charmed. As a result, the patients and their families often do not recognise the abnormal phenomena as mental illnesses, but will seek treatment from traditional healers or temple priests who often reinforce these beliefs. Salleh (1989) showed that $73 \%$ of Malay psychiatric out-patients had sought traditional healers, compared with only $25 \%$ of general medical out-patients. Although this study has been conducted in a less developed part of Malaysia, we still often see patients who have sought traditional healers before seeking psychiatric help.

It is possible that patients may be compliant with the treatment prescribed by a traditional healer, may relabel their experiences as abnormal according to their own set of beliefs, and yet may not see themselves as having a mental illness, or that psychiatric medication is necessary. To the psychiatrist, it is possible to interpret these beliefs as delusional, and patients as lacking in insight, unless they take into account their cultural and ethnic beliefs. It may be unjust to label the patient as lacking in insight, as he does have the insight to seek help from the traditional healer.

It may be possible to apply the components of insight, as advocated by David (1990), to the patients of different cultures, albeit with some allowance. Treatment compliance may be interpreted as either to traditional treatment, or to psychiatric treatment; awareness of illness may be modified to awareness of suffering, and relabelling of psychotic experiences as appropriate to the patient's ethnic and cultural beliefs. This is possible if one has an understanding of the patients' different beliefs. However, I would be rather hesitant to apply his schedule strictly in assessing insight in these patients.

The authors have stressed an indirect approach to help the patient recognise his/her illness so as to improve treatment compliance, rather than tackling non-compliance head on which can only lead 
to antagonism and stalemate. In the treatment of Singaporean patients, we determine whether they sought traditional healers (often they would volunteer this information). While we do not forbid them from continuing to do so, nor disagree with their beliefs, we do explain to them the concept of the Western model of mental illness, so as to improve compliance with psychiatric medication.

DAvid, A. S. (1990) Insight and psychosis. British Journal of Psychiatry, 156, 798-808.

SALLEH, M. R. (1989) The consultation of traditional healers by Malay patients. Medical Journal of Malaysia, 44, 3-13.

WING, J. K., COOPER, J. E. \& SARTORIUS, N. (1974) Measurement and Classification of Psychiatric Symptoms. Cambridge: Cambridge University Press.

Department of Psychological Medicine

National University Hospital

Lower Kent Ridge Road

Singapore 0511

\section{Clozapine and NMS}

SIR: Some patients given neuroleptics after recovering from neuroleptic malignant syndrome (NMS) have not experienced a recurrence of the syndrome (Meltzer, 1973; Rosebush et al, 1989; Pope et al, 1991), although others have (Buckley et al, 1991). The risk of recurrence of NMS may be reduced by allowing two weeks between the episode of NMS and the reintroduction of neuroleptics, by the gradual titration of neuroleptic dosage, and by termination with early signs of a recurrence. The choice of neuroleptic drug is less clear, and rechallenge with the same typical neuroleptic drug, or an agent of a different chemical structure, appears unrelated to the risk of recurrence of NMS (Buckley et al, 1991).

While the recent report by Weller \& Kornhuber (Journal, December 1992, 161, 855-856) concerning the absence of an NMS recurrence in eight of their nine patients treated with clozapine as a rechallenge agent is encouraging, it is nevertheless premature to suggest that NMS, by itself, provides sufficient clinical indication for clozapine therapy. This is not significantly better than the results in other series (Rosebush et al, 1989; Pope et al, 1991). The other significant side-effects associated with clozapine therapy for treatment-refractory schizophrenia especially agranulocytosis (Meltzer, 1992), the ability of clozapine itself to induce NMS, and the potential for diagnostic confusion between fever, hypo- or hypertension, tachycardia, and comparable effects in NMS - suggest that an episode of NMS is not a sufficient reason to justify a trial of clozapine. Treatment-resistance and intolerance of any neuroleptic drug (e.g. severe tardive dyskinesia or dystonia) are the key indications in schizophrenia for clozapine therapy.

Buckley, P., Freyne, A., McCarthy, A., et al (1991) Neuroleptic malignant syndrome - a follow-up study. Irish Journal of Medical Science, 160, 45-47.

MeLtZER, H. Y. (1973) Rigidity, hyperpyrexia and coma following fluphenazine enthanate. Psychopharmacologia, 29, 337-346.

- (1992) Dimensions of outcome with clozapine. British Journal of Psychiatry, 160 (suppl. 17), 22-29.

Pope, H. G. JR, Aizley, H. G., KeCK, P. E. JR, et al (1991) Neuroleptic malignant syndrome: long-term follow-up of 20 cases. Journal of Clinical Psychiatry, 52, 208-212.

Rosebush, P. I., STEwart, T. D. \& GelenberG, A. J. (1989) Twenty neuroleptic rechallenges after neuroleptic malignant syndrome in 15 patients. Journal of Clinical Psychiatry, 50, 295-298.

\section{Peter F. BuCKLeY} Herbert Y. MELTZER

Department of Psychiatry

Case Western Reserve University

2040 Abington Road

Cleveland, Ohio 44106, USA

\section{In defence of clozapine}

SIR: I feel that Dr Healy's excellent and thoughtprovoking 'Devil's advocate' piece about clozapine should not pass without a few further comments to add to Drs McKenna \& Bailey's cogent defence (Journal, January 1993, 162, 23-29 and 32-37 respectively). In condemning clozapine, Dr Healy uses some misplaced logic and reinterpretation of published findings.

Firstly, the comparisons with other treatments are not really relevant. Coronary artery bypass surgery is no longer a dilemma. It was introduced before the advent of calcium-channel blockers and orally absorbable long-acting nitrates and indeed, for a while, was an expensive but realistic option for treatment. With the advent of pharmacological alternatives, no cardiologist would advocate such expensive treatment in advance of cheaper drug treatment, unless there was an immediate lifethreatening indication (e.g. main stem disease). Similarly, the analogy with newer oncological drugs is misplaced. The circumstances surrounding their use means, unlike clozapine, they are essentially untested treatments often tried as a last resort. At best, they may go through open familiarisation trials in very sick patients. It is usually late on in the drugs' lifespan that they would go through rigorous testing.

Perhaps more meaningful comparisons would be with a 'budget-busting' drug such as cyclosporin, 